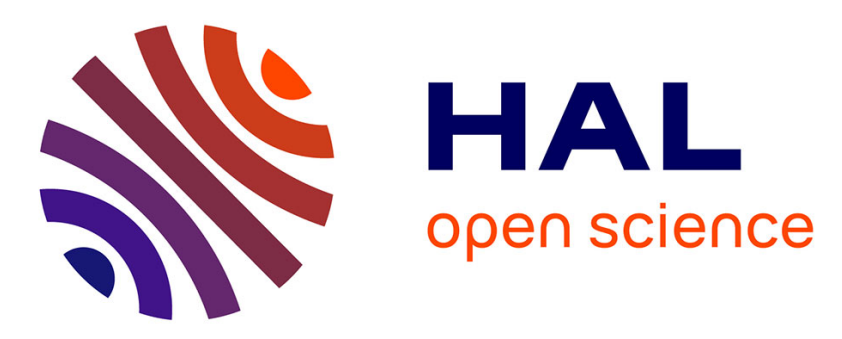

\title{
Nature of soliton interaction in fiber lasers with continuous external optical injection
}

Andrey Komarov, Konstantin Komarov, Alioune Niang, François Sanchez

\section{To cite this version:}

Andrey Komarov, Konstantin Komarov, Alioune Niang, François Sanchez. Nature of soliton interaction in fiber lasers with continuous external optical injection. Physical Review A: Atomic, molecular, and optical physics [1990-2015], 2014, 89 (1), pp.013833. 10.1103/PhysRevA.89.013833 . hal03204340

\section{HAL Id: hal-03204340 \\ https://univ-angers.hal.science/hal-03204340}

Submitted on 21 Apr 2021

HAL is a multi-disciplinary open access archive for the deposit and dissemination of scientific research documents, whether they are published or not. The documents may come from teaching and research institutions in France or abroad, or from public or private research centers.
L'archive ouverte pluridisciplinaire HAL, est destinée au dépôt et à la diffusion de documents scientifiques de niveau recherche, publiés ou non, émanant des établissements d'enseignement et de recherche français ou étrangers, des laboratoires publics ou privés. 


\title{
Nature of soliton interaction in fiber lasers with continuous external optical injection
}

\author{
Andrey Komarov,,${ }^{1,2, *}$ Konstantin Komarov, ${ }^{1}$ Alioune Niang, ${ }^{3}$ and François Sanchez ${ }^{3}$ \\ ${ }^{1}$ Institute of Automation and Electrometry, Russian Academy of Sciences, Acad. Koptyug Pr. 1, 630090 Novosibirsk, Russia \\ ${ }^{2}$ Novosibirsk State Technical University, K. Marx Pr. 20, 630092 Novosibirsk, Russia \\ ${ }^{3}$ Laboratoire de Photonique d'Angers EA 4644, Université d'Angers, 2 Bd. Lavoisier, 49000 Angers, France
}

(Received 19 November 2013; published 24 January 2014)

\begin{abstract}
It has been shown by numerical simulation that the nonlinear interaction between a laser soliton and an injected monochromatic cw results in their phase locking. As a consequence, the velocity of the soliton begins to depend on the amplitude and frequency of the injected radiation. It has been found that if the frequency of the external signal coincides with the frequency of the dispersive waves emitted by solitons in a laser cavity with lumped intracavity elements, a mechanism for controlling long-range soliton interaction occurs. This mechanism is related to the interference between the injected wave and the dispersive waves involved in the strong long-range interaction between solitons. We have demonstrated the mechanism of soliton-soliton repulsion and, as its consequence, the occurrence of harmonic passive mode locking (multipulse generation with an equidistant arrangement of identical solitons in the laser cavity).
\end{abstract}

DOI: 10.1103/PhysRevA.89.013833

PACS number(s): 42.65.Tg, 42.65.Sf, 42.60.Fc

\section{INTRODUCTION}

Stable self-localized waves called solitons are one of the most fascinating nonlinear phenomena. They arise in many fields of physics, including nonlinear optics, hydrodynamics, plasma physics, superfluidity, and so on [1-3]. Among the various nonlinear systems in which such waves occur, passive mode-locked fiber lasers occupy a special place in studies of soliton properties. The nonlinear dispersion parameters of fiber lasers can be varied over a very wide range, which provides a distinct manifestation of these properties. Multisoliton passive mode locking is a common mode of operation of fiber lasers using the nonlinear polarization rotation technique or saturable absorbers [4-10]. The interaction between solitons plays a crucial role in the stable multipulse generation mode. In the case of soliton attraction, the states of bound solitons or soliton crystals can occur [11-19]. Repulsive interaction between solitons is responsible for harmonic passive mode locking [20,21]. In many fiber lasers with harmonic passive mode locking, a narrow-band spectral component of the radiation has been observed [22-27]. It would appear reasonable that this component may play an important role in the mechanism resulting in harmonic mode locking. Indeed, it has been shown experimentally [28] that continuous external optical injection into a passively mode-locked Er:Yb-doped fiber laser leads to harmonic mode locking. The effect of this injection on the motion of solitons relative to each other in the fiber laser has been analyzed in [29]. It has been found that the soliton motion is affected by the injected cw in such a manner that a soliton gas or liquid or crystal can be obtained depending on the intensity and frequency of the injected radiation.

In this paper we investigate the nature of the mechanism of soliton-soliton repulsion resulting in harmonic passive mode locking of a fiber laser with continuous external optical injection. In Sec. II we analyze the interaction of an injected $\mathrm{cw}$ with an individual soliton using the model of a distributed intracavity nonlinear medium. Section III deals with the soliton

*Corresponding author: komarov@iae.nsk.su interaction and the mechanism of harmonic passive mode locking in a fiber laser with lumped nonlinear losses due to the nonlinear polarization rotation technique. In this case, the long-range interaction between solitons involves the dispersive waves emitted by solitons because of their periodic interaction with intracavity lumped elements. Section IV is devoted to the discussion of the results obtained.

\section{MODEL OF AN UNIFORMLY DISTRIBUTED INTRACAVITY MEDIUM}

In the first stage of our analysis, we use the normalized complex dispersion equation with a cubic-quintic nonlinearity which describes the field evolution in a unidirectional ring laser with a uniformly distributed intracavity medium [30-32]:

$$
\begin{aligned}
\frac{\partial E}{\partial \zeta}= & \left(D_{r}+i D_{i}\right) \frac{\partial^{2} E}{\partial \tau^{2}}+\left[g+(p+i q) I-\left(p_{2}+i q_{2}\right) I^{2}\right] E \\
& +P \exp (i \delta \omega \tau-i \delta \kappa \zeta),
\end{aligned}
$$

where $E$ is the electric field amplitude, $\tau$ is the time coordinate in units $\delta t=\sqrt{\left|\beta_{2}\right| L / 2}$ (here $\beta_{2}$ is the second-order groupvelocity dispersion for the intracavity medium and $L$ is the cavity length), $\zeta$ is the normalized propagation distance (the number of passes of the radiation through the laser cavity), $D_{r}$ is the frequency dispersion of the gain and the linear losses, $D_{i}$ is the frequency dispersion of the refractive index, $p$ and $p_{2}$ are the cubic and quintic nonlinearities of the losses $(p>0$, $\left.p_{2}>0\right), q$ and $q_{2}$ are the cubic and quintic nonlinearities of the refractive index, and $I=|E|^{2}$ is the field intensity in units $(\gamma L)^{-1}$, where $\gamma\left(\mathrm{W}^{-1} \mathrm{~m}^{-1}\right)$ is the dimensional nonlinear refractive coefficient related to the nonlinear refractive index coefficient [5]. The term $g$ is the total amplification including the linear losses $\sigma_{0}$ :

$$
g=\frac{a}{1+b \int I d \tau}-\sigma_{0},
$$

where the integration is carried out over the whole round-trip period, $a$ is the pumping parameter, and $b$ is the saturation parameter. The last term in Eq. (1) describes the electric-dipole 
polarization of the intracavity medium that produces the monochromatic radiation inside the laser cavity. In this way, we model the injected radiation inside the laser cavity. The parameters $\delta \omega$ and $\delta \kappa$ are determined by the frequency and wave vector of the external injected monochromatic radiation. Here $\delta \omega$ is the detuning of the carrier frequency of the external radiation from the center of the spectral gain band. The parameters $\delta \omega$ and $\delta \kappa$ are chosen so that the injected radiation coincides with the radiation of one of the longitudinal modes of the laser resonator. This implies that $\delta \kappa$ and $\delta \omega$ satisfy the dispersion relation

$$
\delta \kappa=D_{i}(\delta \omega)^{2}
$$

which is obtained from Eq. (1). In addition, for this purpose in our numerical simulation, we choose the frequency detuning in the form $\delta \omega=2 \pi K / l$, where the frequency parameter $K$ is an integer and $l$ is the length of the temporal computation box. Varying the parameter $P$, we can change the intensity $I_{\mathrm{cw}}$ of the intracavity injected cw. Equation (1) takes into account the gain saturation through the dependence of the parameter $g$ on the energy of the intracavity radiation [see Eq. (2)]. This differs from the equation used in $[31,33]$, where the parameter $g$ is a constant.

Using Eqs. (1) and (2) we performed our numerical simulation of laser passive mode locking. The parameters of these equations are chosen to be similar to the parameters of a passively mode-locked Er-doped laser: $a=0.3, b=0.01$, $D_{r}=0.2, D_{i}=1, \sigma_{0}=0.5, p=1, p_{2}=0.1, q=1$, and $q_{2}=0$. An anomalous frequency dispersion regime $D_{i}>$ 0 is investigated. Without optical injection, the phase of the steady-state soliton established after a transient process increases linearly with increasing propagation distance $\zeta$. Similarly, without any soliton, the phase of the injected cw also increases linearly with increasing $\zeta$. When these rates of the phase increase coincide or are close to each other, resonant interaction between the soliton and the cw occurs. This interaction results in phase locking of the soliton and the cw. In this case, the phases of the field at all points of laser cavity have the same linear dependence on the propagation distance $\zeta$. Figure 1 shows the boundary values of the detuning parameter $K$ for which phase locking occurs for various levels of the cw intensity $I_{\mathrm{cw}}$ inside the laser resonator. The obtained

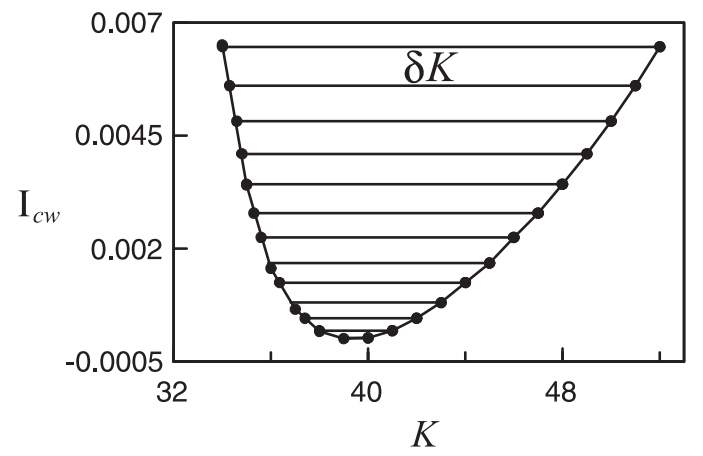

FIG. 1. Phase-locking region of a single soliton and an injected $\mathrm{cw}$ in the plane $I_{\mathrm{cw}}$ (intracavity intensity of the $\mathrm{cw}$ ), $K$ (frequency detuning parameter of the $\mathrm{cw}$ from the center of the spectral gain band) for the distributed model described by Eq. (1).

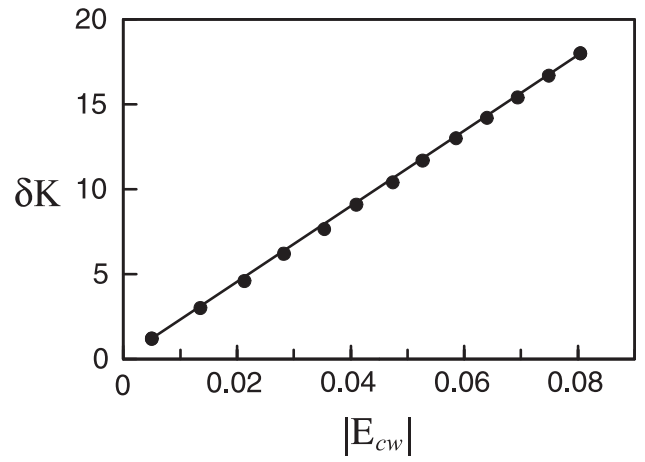

FIG. 2. Dependence of the frequency interval $\delta K$ of the phase locking of a single soliton and an injected $\mathrm{cw}$ on the intracavity $\mathrm{cw}$ amplitude $\left|E_{\mathrm{cw}}\right|$.

boundary curve corresponds to the minimal values of $P$ for which phase locking still exists. As one can see from Fig. 2, the width of the phaselocking interval $\delta K$ depends linearly on the amplitude of the intracavity $\mathrm{cw}\left|E_{\mathrm{cw}}\right|$ for its small values.

In the case of phase locking of a steady-state soliton and an injected cw, the phase difference for any two points of the united field structure is constant. The united structure moves as a whole. As a result, the velocity of the soliton depends on the parameters of the injected $\mathrm{cw}$. Figure 3 demonstrates the change in the soliton velocity $\delta v=d \tau / d \zeta$ with a change in the detuning parameter $K$ due to the asymmetry of the soliton pedestal that arises [34]. A large change in the soliton velocity is observed in the phase-locking region (values of $K$ between points 1 and 2 in Fig. 3). Here the soliton motion is translational. Outside the phase-locking region, the phase difference oscillates. As a consequence, the structure of the soliton wings changes periodically. As a result, the soliton motion becomes oscillatory translational and the change in the averaged soliton velocity becomes small.

The laser model with a uniformly distributed intracavity medium is sufficiently simple and adequately describes many features of passive mode locking. However, this model is suitable only for short-range interaction between solitons with an interaction range equal to several soliton lengths. It is not

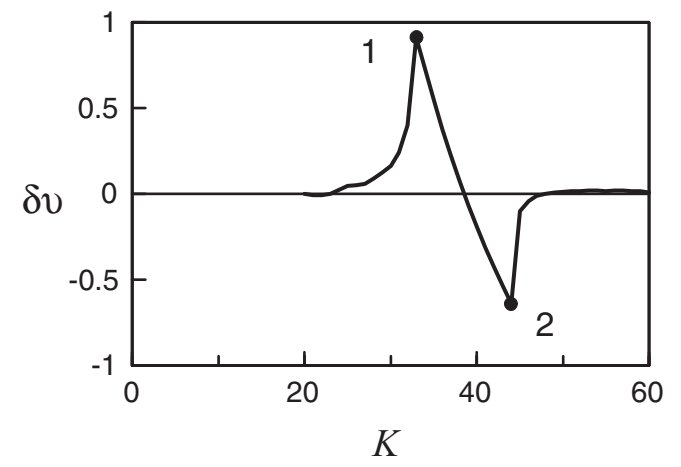

FIG. 3. Change in the soliton velocity $\delta v$ vs the frequency detuning $K$ of the $\mathrm{cw}$ from the center of the spectral gain band. The spectral interval between points 1 and 2 corresponds to the phase locking of the single soliton and the injected $\mathrm{cw}$. 
suited for investigation of the long-range interaction due to the dispersive waves caused by lumped intracavity elements [35].

\section{LASER WITH THE NONLINEAR POLARIZATION ROTATION TECHNIQUE}

The soliton circulating in the laser cavity periodically experiences perturbations caused by lumped nonlinear losses and various intracavity components. After each perturbation, the soliton emits a dispersive wave. Constructive interference between these waves forms powerful spectral sidebands and powerful extended soliton wings [36]. These wings result in long-range interaction, providing the formation of bound steady states of interacting solitons with a large binding energy [35]. In fiber lasers with the nonlinear polarization rotation technique, the nonlinear losses are essentially lumped.

For our analysis of the role of continuous external optical injection in the interaction between solitons in a fiber laser with the nonlinear polarization rotation technique, we use the following equations $[5,37]$ :

$$
\begin{aligned}
\frac{\partial E_{x}}{\partial \zeta}= & \left(D_{r}+i D_{i}\right) \frac{\partial^{2} E_{x}}{\partial \tau^{2}}+g E_{x}+i q\left(\left|E_{x}\right|^{2} E_{x}\right. \\
& \left.+A\left|E_{y}\right|^{2} E_{x}+B E_{y}^{2} E_{x}^{*}\right)+P \exp (i \delta \omega \tau-i \delta \kappa \zeta),
\end{aligned}
$$

$$
\begin{aligned}
\frac{\partial E_{y}}{\partial \zeta}= & \left(D_{r}+i D_{i}\right) \frac{\partial^{2} E_{y}}{\partial \tau^{2}}+g E_{y} \\
& +i q\left(\left|E_{y}\right|^{2} E_{y}+A\left|E_{x}\right|^{2} E_{y}+B E_{x}^{2} E_{y}^{*}\right),
\end{aligned}
$$

where $E_{x}$ and $E_{y}$ are two orthogonal components of the electric field, $A=2 / 3$ and $B=1 / 3$, here $I=\left|E_{x}\right|^{2}+\left|E_{y}\right|^{2}$, and the gain $g$ is determined by Eq. (2). Equations (4) and (5) describe the field evolution in the fiber of a unidirectional ring fiber laser. The laser resonator contains a polarization control system including the following sequentially arranged components: a half-wave phase plate with orientation angle $\alpha_{3}$ with respect to the $x$ axis, a quarter-wave plate (orientation angle $\alpha_{3}$ ), a polarizing isolator (the passing axis is parallel to the $x$ axis), and a second quarter-wave plate (orientation angle $\alpha_{1}$ ) [5]. The polarization control system produces nonlinear losses that form ultrashort pulses in the laser resonator. As in the case of Eq. (1), the last term in Eq. (4) describes the electric-dipole polarization of the intracavity medium which induces the injected monochromatic $\mathrm{cw}$ inside the laser cavity. For our numerical simulation, the parameters of these equations are chosen to be close to parameters of the passively mode-locked Er:Yb-doped laser investigated in [28]. We use the following parameters for the system analyzed: $a \approx 0.5, b=0.02, \sigma_{0}=$ $0, D_{r}=0.1, D_{i}=0.7, q=1, \alpha_{1}=0.2, \alpha_{2}=0.4, \alpha_{3}=-0.2$, and $l \approx 164$.

Figure 4(a) shows the soliton pedestal with powerful wings dueto dispersive waves which occurs after a transient process. Here an injected $\mathrm{cw}$ is absent $(P=0)$. At the distant wings, the intensity $I(\tau)$ approaches zero. The radiation distribution $I(\tau)$ is repeated with a round-trip period $\delta \zeta=1$. The oscillations of the intracavity field with this period are due to the interaction of the soliton with the lumped intracavity elements. In the case of a uniformly distributed intracavity medium, such
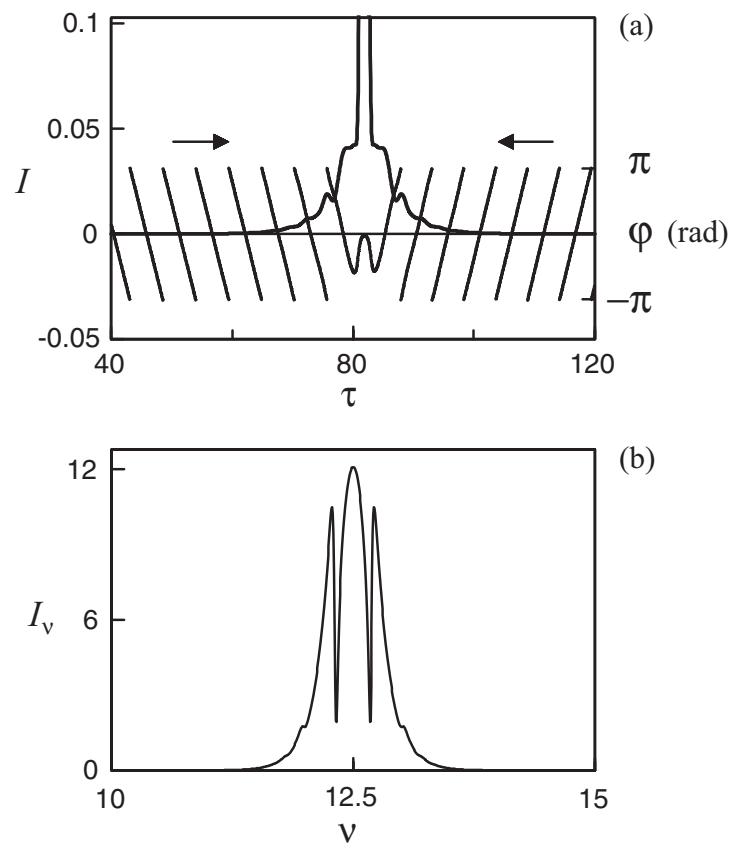

(b)

FIG. 4. (a) Distributions of the soliton intensity $I(\tau)$ and the phase $\varphi(\tau)$ in the vicinity of the soliton pedestal without external optical injection $(P=0)$. (b) Spectral distribution of the soliton.

oscillations are absent. For the laser parameters used here, the phase of the field at any point of the space increases by the same positive value each round-trip period. As a result, at the distant wings of the pulse, points with a certain fixed phase move to the center of the pulse. The directions of this motion are given by arrows in Fig. 4(a). The phase difference for any two points of the pulse is constant from one round trip to the next. The powerful soliton wings result in the powerful spectral sidebands shown in Fig. 4(b). The peaks of these sidebands are formed by dispersive waves for which they are phase locked to the soliton. The fulfillment of the phase-locking condition results from the stationarity of the phase difference for any two points of the field distribution, one of which is in the central part of the soliton and the other at its wing.

Figure 5 shows the steady-state distributions of the intensity $I(\tau)$ and the spectrum in the case of an injected $\mathrm{cw}$ in the laser cavity. At the distant wings of the soliton, the intensity $I(\tau)$ approaches the intensity $I_{\mathrm{cw}}$ of the injected $\mathrm{cw}$. The radiation distribution $I(\tau)$ is repeated with a round-trip period $\delta \zeta=1$. The phase difference for any two points of the field distribution is also constant, implying phase locking of the soliton and the intracavity injected cw. In this case, the frequency parameter $K$ of the injected $\mathrm{cw}$ corresponds to the peak of the right frequency sideband [see Fig. 5(b)]. This implies that the frequency parameter of the right soliton wing $K_{\mathrm{rw}}$, due to dispersive waves, coincides with the corresponding parameter of the injected cw $K_{\mathrm{rw}}=K$. Accordingly, for the frequency parameter of the left soliton wing $K_{\mathrm{lw}}$, we have $K_{\mathrm{lw}}=-K_{\mathrm{rw}}=$ $-K$. An analysis of the phase distributions [Figs. 4(a) and 5(a)] and the intensity distribution presented in Fig. 5(a) shows the following. The phase of the center of the soliton coincides with the phase of the injected $\mathrm{cw}$ at the point of location of the center of the soliton. The injected cw and the wave due to 

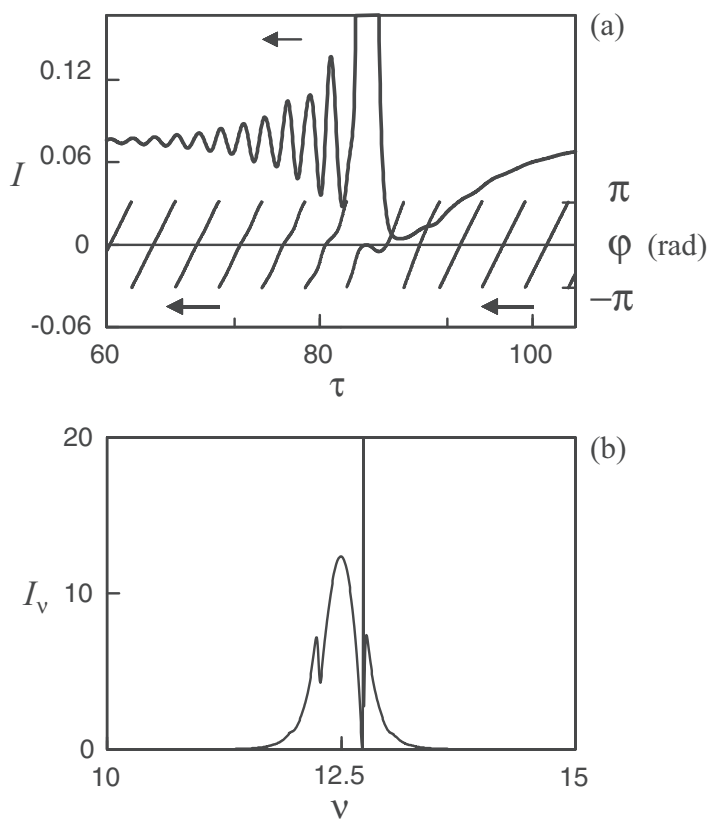

FIG. 5. (a) Distributions of the intensity $I(\tau)$ and phase $\varphi(\tau)$ of the field in the vicinity of the soliton pedestal in the case of an injected cw $(P \neq 0)$. (b) Spectral distribution of the resulting radiation.

the right soliton wing have the same frequency parameter $K$ and the same phase velocity, move in the same direction, and lead to destructive interference which decreases the resulting wave field at the right wing of the soliton [see Fig. 5(a)]. The injected $\mathrm{cw}$ and the wave due to the left soliton wing have frequency parameters and phase velocities that are equal in value but opposite in sign, move in opposite directions, and form a standing wave. At the maxima of the standing wave [see Fig. 5(a)], these waves have the same phase and here constructive interference of the waves occurs. At the minima of the standing wave, the waves have a phase difference equal to $\pi$ and here destructive interference occurs.

Figure 6 shows the dependence of the soliton velocity on the intensity $I_{\mathrm{cw}}$ of the injected cw. Phase locking of the soliton and the injected cw occurs in the intensity interval between points 1 and 3 . Below point 1 , phase locking is absent. Above point 3 , the injected cw destroys the single soliton operation. Between

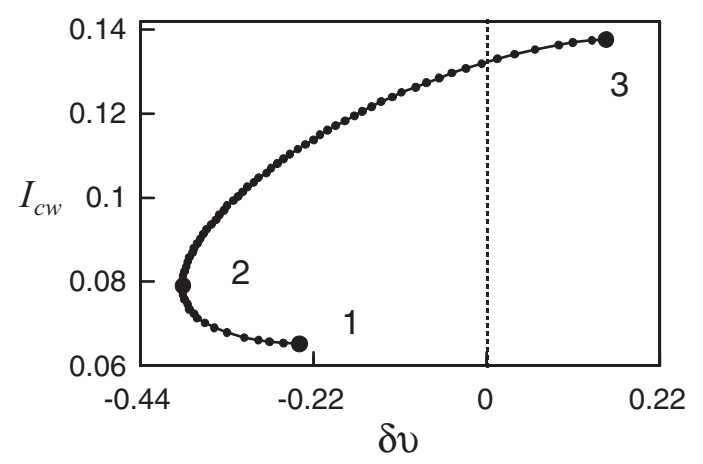

FIG. 6. Soliton velocity $\delta v$ vs the intensity $I_{\mathrm{cw}}$ of the injected cw. $K=40$.

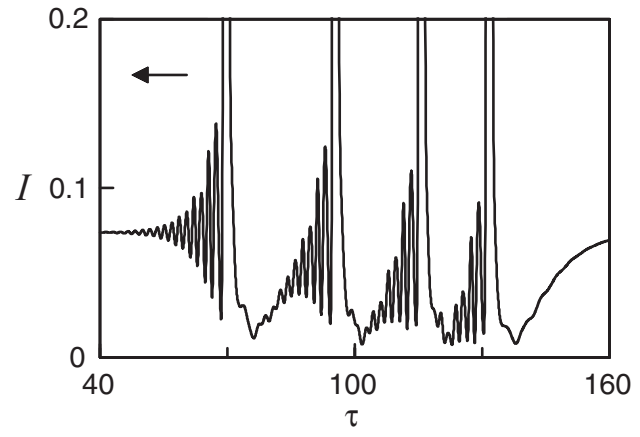

FIG. 7. Distribution of the intensity $I(\tau)$ in a train of solitons. The arrow shows the direction of motion of the solitons due to the injected cw. $K=40$.

points 1 and 2 and between points 2 and 3, the dependence of $\delta v$ on $I_{\mathrm{cw}}$ is monotonic.

Figure 7 demonstrates the repulsion mechanism of laser solitons. After each pulse, the injected $\mathrm{cw}$ attenuates because of its destructive interference with the right-wing dispersive waves. As a consequence, the resultant $\mathrm{cw}$ decreases monotonically from left to right along the pulse train (see Fig. 7). The changing intensity of the resultant $\mathrm{cw}$ is between points 1 and 2 in Fig. 6, where the velocity of motion of pulses in the left direction decreases monotonically with decreasing intensity $I_{\mathrm{cw}}$. As a result, the velocity of the solitons motion in the left direction decreases monotonically along the soliton train from left to right. This distribution of soliton velocities in the train results in an increase of intersoliton spacing, that is, to repulsion of the solitons. Figure 8 shows the repulsion of initially closely spaced bound solitons and the realization of the harmonic passive mode locking (multipulse operation with equidistant pulses) occurring as a result of this repulsion after a transient process. In the steady-state lasing regime, all pulses have the same phases at the points of their peak amplitudes. For the laser parameters used, the duration of the transient process is approximately equal to 8000 round-trip periods. In the steady-state regime of harmonic passive mode locking, the ratio of the intersoliton interval to the soliton

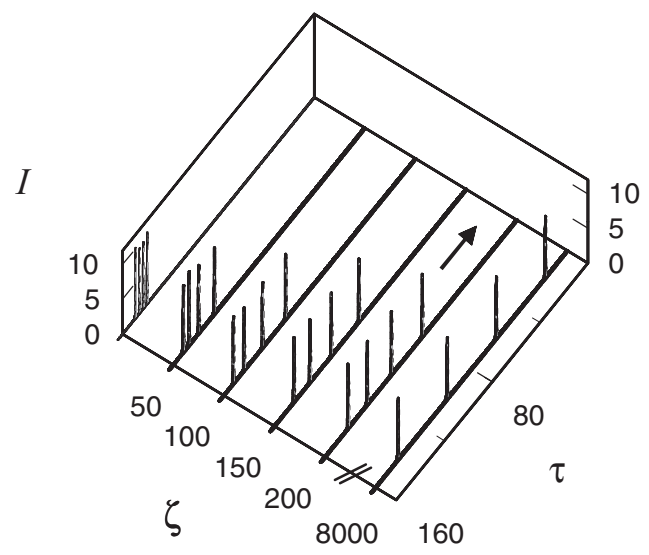

FIG. 8. Dependence of the intensity distribution $I(\tau)$ on the number of round trips $\zeta$ in a passive mode-locked laser with an injected cw. $K=40$. 
width is approximately equal to 70 . In the case of three-pulse operation, it is equal to 100 .

We have also observed an attraction of solitons. In the case of the frequency parameter outward from $K=40$, we have observed a soliton attraction for great intersoliton distances and their repulsion for short ones. As a consequence, the usual regime of bound solitons has been realized.

\section{DISCUSSION}

We started from the complex dispersion master equation with cubic-quintic nonlinearity which describes the field evolution in a laser with a uniformly distributed intracavity medium. An external injected cw effectively influences the motion of the soliton in the case of their resonant interaction which induces their phase locking. Previously similar phase locking was considered in [38] within the framework of the nonlinear Schrödinger equation describing an electromagnetic interaction in a two-fiber coupler with soliton in one fiber and $\mathrm{cw}$ in another. We have found that in a fiber laser the frequency detuning range for the injected $\mathrm{cw}$ in which phase locking occurs is proportional to its amplitude. The resulting change in the soliton velocity depends on the intensity of the injected cw and its frequency detuning. The model with a uniformly distributed intracavity medium describes only the short-range interaction between solitons. In the next step, we have investigated a model with lumped nonlinear losses due to the nonlinear polarization rotation technique.

Because of the periodic interaction of the soliton with lumped intracavity elements, it emits dispersive waves. The dispersive waves resonantly interact with the soliton to form its powerful extended wings. These wings are responsible for the long-range interaction between solitons. The large extended wings of the soliton form large narrow sidebands in its spectrum. This results in the following features in the interaction of the injected $\mathrm{cw}$ with an individual soliton and the interaction between solitons. First, the resonant interaction of the injected cw with the soliton occurs only if the frequency detuning of the $\mathrm{cw}$ falls within the spectral sideband of the soliton. Second, the resonant interaction of the injected $\mathrm{cw}$ and the dispersive soliton wing produces a resultant wave which modifies the motion of other solitons. Thus, we can control the long-range interaction between solitons through the injected cw. In our numerical experiment, we have demonstrated the transition from the lasing regime of bound solitons to harmonic passive mode locking due to an injected $\mathrm{cw}$. This transition has also been observed in experimental studies of a passively mode-locked Er:Yb-doped fiber laser with continuous external optical injection. It occurs by repulsive interaction between solitons. In our numerical simulation, we have demonstrated the mechanism of this interaction. The injected $\mathrm{cw}$ and the dispersive wing of the first soliton form a destructive interference, resulting in a monotonic change in the intensity of the resultant cw along the fiber cavity. This resultant cw changes the velocity of the second soliton, leading to repulsion between these solitons. In our numerical experiment, the distance of the repulsive interaction was about 100 lengths of an individual soliton, which is consistent with the experimental data of [28]. As in that study, the frequency detuning range where harmonic passive mode locking takes place is narrow (about $1 \%$ of the spectral gain bandwidth). In addition, the model predicts a threshold value for the injected power above which harmonic passive mode locking occurs. This is also consistent with experimental results reported in [28].

The next step in the theoretical description of the features of soliton interaction will be a study of the case with a nonmonotonically changing resultant $\mathrm{cw}$. In this case, it is possible to expect the generation of soliton trains of fixed length and also the generation of a train with a fixed intersoliton spacing in the train. In addition, the constructive interference of the injected cw and the soliton dispersive wing may enhance the resultant cw, which may also lead to new interesting lasing features and requires a further study.

In this paper we have investigated the interaction between laser solitons through the dispersive waves connected with a periodic modulation of nonlinear losses. In [39] it was shown that the modulation of a dispersion of a fiber refractive index also results in a long-range soliton interaction. Such mechanism of an interaction can be realized in the laser with several fibers with different dispersions. The peculiarities of the interaction between solitons in this case demand a special study. The peculiarities connected with a finiteness of a spectral width of injected radiation, with a variation of its polarization characteristics, and with an injection of a biharmonic signal also demand a special investigation.

\section{ACKNOWLEDGMENTS}

The work was supported by the Agence Nationale de la Recherche (Contract No. ANR-2010-BLANC-0417-01SOLICRISTAL) and the RF President's grant, Grant No. NS-6170.2012.2.
[1] M. Remoissenet, Waves Called Solitons (Springer-Verlag, Berlin, 1994).

[2] N. N. Akhmediev and A. Ankiewicz, Solitons (Chapman and Hall, London, 1997).

[3] I. S. Aranson and L. Kramer, Rev. Mod. Phys. 74, 99 (2002).

[4] D. Y. Tang, L. M. Zhao, B. Zhao, and A. Q. Liu, Phys. Rev. A 72, 043816 (2005).

[5] A. Komarov, H. Leblond, and F. Sanchez, Phys. Rev. A 71, 053809 (2005).

[6] A. K. Komarov and K. P. Komarov, Phys. Rev. E 62, R7607 (2000).
[7] H. Zhang, D. Y. Tang, L. M. Zhao, Q. Bao, and K. Loh, Opt. Commun. 283, 3334 (2010).

[8] C. S. Jun, J. H. Im, S. H. Yoo, S. Y. Choi, F. Rotermund, D.-I. Yeom, and B. Y. Kim, Opt. Expess 19, 19775 (2011).

[9] X. Wu, D. Y. Tang, X. N. Luan, and Q. Zhang, Opt. Commun. 284, 3615 (2011).

[10] J.-L. Xu, X.-L. Li, Y.-Z. Wu, X.-P. Hao, J.-L. He, and K.-J. Yang, Opt. Lett. 36, 1948 (2011).

[11] B. A. Malomed, Phys. Rev. A 44, 6954 (1991).

[12] Ph. Grelu, F. Belhache, F. Gutty, and J. M. Soto-Crespo, Opt. Lett. 27, 966 (2002). 
[13] V. V. Afanasjev, B. A. Malomed, and P. L. Chu, Phys. Rev. E 56, 6020 (1997).

[14] Ph. Grelu, J. Béal, and J.-M. Soto-Crespo, Opt. Express 11, 2238 (2003).

[15] X. Liu, Phys. Rev. A 81, 023811 (2010).

[16] A. Komarov, K. Komarov, and F. Sanchez, Phys. Rev. A 79, 033807 (2009).

[17] X. Liu, Phys. Rev. A 84, 053828 (2011).

[18] F. Amrani, A. Haboucha, M. Salhi, H. Leblond, A. Komarov, and F. Sanchez, Appl. Phys. B 99, 107 (2010).

[19] J. Peng, L. Zhan, S. Luo, and Q. S. Shen, IEEE Photonics Technol. Lett. 25, 948 (2013).

[20] A. B. Grudinin and S. Gray, J. Opt. Soc. Am. B 14, 144 (1997).

[21] B. Fu, L. Gui, W. Zhang, X. Xiao, H. Zhu, and C. Yang, Opt. Commun. 286, 304 (2013).

[22] F. Amrani, A. Haboucha, M. Salhi, H. Leblond, A. Komarov, Ph. Grelu, and F. Sanchez, Opt. Lett. 34, 2120 (2009).

[23] G. Sobon, K. Krzempek, P. Kaczmarek, K. M. Abramski, and M. Nikodem, Opt. Commun. 284, 4203 (2011).

[24] Z. X. Zhang, L. Zhan, X. X. Yang, S. Y. Luo, and Y. X. Xia, Las. Phys. Lett. 4, 592 (2007).

[25] F. Amrani, A. Niang, M. Salhi, H. Leblond, and F. Sanchez, Opt. Lett. 36, 4239 (2011).

[26] J.-M. Soto-Crespo, N. Akhmediev, Ph. Grelu, and F. Belhache, Opt. Lett. 28, 1757 (2003).
[27] A. Komarov, K. Komarov, H. Leblond, and F. Sanchez, J. Opt. A: Pure Appl. Opt. 9, 1149 (2007).

[28] A. Niang, F. Amrani, M. Salhi, H. Leblond, A. Komarov, and F. Sanchez, Opt. Commun. 312, 1 (2014).

[29] H. Leblond, A. Niang, F. Amrani, M. Salhi, and F. Sanchez, Phys. Rev. A 88, 033809 (2013).

[30] K. P. Komarov, Opt. Commun. 54, 233 (1985).

[31] N. N. Akhmediev, A. Ankiewicz, and J.-M. Soto-Crespo, Phys. Rev. Lett. 79, 4047 (1997).

[32] A. K. Komarov, K. P. Komarov, and F. M. Mitschke, Phys. Rev. A 65, 053803 (2002).

[33] W. Chang, J.-M. Soto-Crespo, A. Ankiewicz, and N. N. Akhmediev, Phys. Rev. A 79, 033840 (2009).

[34] A. Komarov and F. Sanchez, Phys. Rev. E 77, 066201 (2008).

[35] A. Komarov, F. Amrani, A. Dmitriev, K. Komarov, D. Meshcheriakov, and F. Sanchez, Phys. Rev. A 85, 013802 (2012).

[36] S. M. J. Kelly, Electron Lett. 28, 806 (1992).

[37] D. Y. Tang, B. Zhao, L. M. Zhao, and H. Y. Tam, Phys. Rev. E 72, 016616 (2005).

[38] F. Kh. Abdullaev, S. A. Darmanyan, and P. K. Khabibullaev, Optical Solitons (Springer-Verlag, Heidelberg, 1993).

[39] M. Chertkov, Y. Chung, A. Dyachenko, I. Gabitov, I. Kolokolov, and V. Lebedev, Phys. Rev. E 67, 036615 (2003). 